January 1997

TAUP 2405/97

\title{
BEHAVIOUR OF THE FORWARD PEAK IN HARD DIFFRACTIVE LEPTOPRODUCTION OF VECTOR MESONS
}

\author{
E. G O T S M A N ${ }^{a), 1)}$, E. L E V I N $\mathrm{N}^{a), b), 2)}$ and U. M A O R ${ }^{a), 3)}$ \\ a) School of Physics and Astronomy \\ Raymond and Beverly Sackler Faculty of Exact Science \\ Tel Aviv University, Tel Aviv, 69978, ISRAEL \\ b) Theory Department, Petersburg Nuclear Physics Institute \\ 188350, Gatchina, St. Petersburg, RUSSIA
}

\begin{abstract}
:
The measured forward slope in elastic and inelastic leptoproduction of vector mesons differ by a substantial amount. In an attempt to describe this phenomenon we construct a two radii model for the target proton, and estimate the effective parameters of the hard Pomeron obtained from a pQCD dipole model with eikonal shadowing corrections (SC). We show that the SC reduce the intercept of the hard Pomeron and generate an effective shrinkage of the forward peak and a diffractive dip at $|t| \approx 1 \mathrm{GeV}^{2}$, which appears to affect the value of the experimentally measured slope.
\end{abstract}

\footnotetext{
1) Email: gotsman@post.tau.ac.il .

2) Email: leving@ccsg.tau.ac.il.

3) Email: maor@ccsg.tau.ac.il.
} 


\section{Introduction}

Perturbative QCD (pQCD) calculations of diffractive leptoproduction of vector mesons (DLVM) in deep inelastic scattering (DIS) are based on the observation [1, 2, 3] that DLVM is a hard process, dominated by short transverse distances $r_{\perp}^{2} \propto \frac{1}{Q^{2}}$. In contrast, elastic photoproduction of light vector mesons has the typical properties of soft interactions, i.e. a pronounced forward peak shrinking with energy, and a mild energy dependence of the integrated cross section. The forward peak of DLVM in DIS is less distinct while the energy dependence of the integrated cross section is rather steep [4, (5]. The same properties are seen in real photoproduction of $\mathrm{J} / \psi$, 4 , 5] where $4 m_{\psi}^{2}$ replaces $Q^{2}$ as a measure of the hard scale [1]. These experimental observations justify a pQCD calculation [6, 7, 8] in which $x$ dependence of calculated cross section is determined by $\left[x G\left(x, Q^{2}\right)\right]^{2}$, where $x G\left(x, Q^{2}\right)$ denotes the gluon distribution within the target.

The goal of this letter is to examine the detailed behaviour of the forward DLVM cross section. To this end we shall utilize the expotential approximation for

$$
\frac{d \sigma}{d t}=\left(\frac{d \sigma}{d t}\right)_{t=0} e^{-B|t|},
$$

where the $t=0$ slope is defined by

$$
B=\left.\frac{\frac{d}{d t}\left(\frac{d \sigma}{d t}\right)}{\frac{d \sigma}{d t}}\right|_{t=0}
$$

The properties of the differential cross section in high energy soft hadronic processes are well understood, these are dominated by Pomeron exchange, where we have

$$
\alpha(t)=\alpha_{P}(0)+\alpha_{P}^{\prime} t=1+\epsilon+\alpha_{P}^{\prime} t
$$

The energy dependence of the forward differential cross section is determinated by $\epsilon$, whereas the slope $B=B_{0}+2 \alpha_{P}^{\prime} \ln \left(\frac{s}{s_{0}}\right)$ depends on $\alpha_{P}^{\prime}$. A good reproduction of the hadronic data is obtained using the Regge parameterization [9, 10], with $\epsilon=0.08$ and $\alpha_{P}^{\prime}=0.25 \mathrm{GeV}^{-2}$. The observation that $\alpha_{P}^{\prime} \neq 0$, is associated with the experimentally observed shrinkage of the forward differential elastic cross section, with increasing energy $\left(s=W^{2}\right)$. It is very convenient to parameterize the hard Pomeron in a similar fashion, where $\epsilon\left(Q^{2}\right)$ and $\alpha_{P}^{\prime}\left(Q^{2}\right)$ provide the relevant information on the $W$ and $t$ dependence of the DLVM cross section.

An important property, observed in $p p$ and $\bar{p} p$ elastic scattering in the ISR-Tevatron range, is a diffractive dip of $\frac{d \sigma}{d t}$ positioned at $|t| \approx 1.4 \mathrm{GeV}^{2}$ at the lower ISR energies, 
which moves slowly towards smaller $t$ as the energy is increased. This phenomenon has been associated with screening re-scattering corrections, which are calculated with relative ease in the eikonal approximation [11, 12, 13]. An obvious consequence of a dip at higher

$|t|$ values, is that, the exponential approximation for $\frac{d \sigma}{d t}$ is only valid in the narrow forward cone, as $\mathrm{B}$ acquires a positive curvature outside this cone.

In the following we study the dependence of the forward DLVM cross section on $W, t$ and $Q^{2}$. Specifically we wish to examine:

1) The $Q^{2}$ dependence of $\epsilon$ in hard DLVM.

2) The phenomena of shrinkage and a diffractive dip in the wide forward cone of hard DLVM, as a function of $W$ and $Q^{2}$.

We note that for the hard Pomeron in the DGLAP approach $\epsilon$ grows with $Q^{2}$, and there is no shrinkage or diffractive dips. We re-examine these features following our paper [6] and utilize a screened dipole model in the DGLAP evolution equations, which have been shown to be adequate for the HERA kinematical range. Furthermore, we note that the measured slopes in elastic and inelastic DLVM differ by a substantial amount [14]. This suggests the possibility that there are two intrinsic radii in the target proton. To investigate this possibility, we have constructed an appropriate model with two radii which takes into account the SC. Our calculated results are compared with the available experimental data. We are successful in reproducing the experimental results for $\mathrm{J} / \psi$, however, for $\rho$ production our pQCD calculations do not agree with data. We attribute this deficiency to the presence of a non negligible soft Pomeron component, and discuss it briefly.

\section{Main formulae}

A general approach to the SC for DLVM in DIS, has been developed in Ref. [6], following Refs. [16] [17]. It was shown [6] that the diffractive production of vector mesons occurs at small distances, and hence the passage of the quark - antiquark pair through the target, can be calculated in pQCD utilizing the eikonal approximation. The situation with the SC in the gluon sector turns out to be more complicated. However, in the eikonal approach the SC can be absorbed into gluon density. The general formula for the screened DLVM amplitude [6] is:

$$
A\left(b_{t} ; Q, x\right)=C \int \frac{d^{2} r_{\perp}}{\pi} \int_{0}^{1} d z \Psi^{\gamma^{*}}\left(Q, r_{\perp}, z\right) 2\left\{1-e^{-\kappa\left(r_{\perp}, x ; b_{t}\right)}\right\}\left[\Psi^{V}\left(r_{\perp}=0, x\right)\right]^{*}
$$

where $\Psi^{\gamma^{*}}, \Psi^{V}$ denote the wave functions of the virtual photon and vector meson respectively. The constant $C$ has been calculated in Refs. [1] [3] [6]. $r_{\perp}$ denotes the transverse 
distance between the quark and antiquark, and $z$ the fraction of the photon energy carried by the quark (antiquark). $W$ denotes the energy and $b_{t}$ the impact parameter of the reaction. $x=\frac{Q^{2}+m_{V}^{2}}{W^{2}}$, where $m_{V}$ is the vector meson mass. The degree of the $\mathrm{SC}$ in Eq. (4) is characterized by the parameter $\kappa$ defined below. In our model $\kappa$ depends on the structure of the target.

\subsection{One radius approach.}

In the simplest one radius model for the target structure, we have [6]:

$$
\kappa\left(b_{t} ; r_{\perp}, x\right)=\frac{2 \pi \alpha_{\mathrm{S}} r_{\perp}^{2}}{3} \Gamma\left(b_{t}\right) x G\left(x, \frac{4}{r_{\perp}^{2}}\right) .
$$

Here, we have used the main property of the DGLAP evolution equations, which allows us to factor out the $b_{t}$ dependence from $x$ and $r_{\perp}$ (see Ref. 19]). $\Gamma\left(b_{t}\right)$ denotes the Fourier transform of the two gluon form factor of the target, which has the same form for any process.

The relation between the profile $\Gamma\left(b_{t}\right)$ and the two gluon form factor reads

$$
\Gamma\left(b_{t}\right)=\frac{1}{\pi} \int e^{-i\left(\vec{b}_{t} \cdot \vec{q}_{t}\right)} F(t) d^{2} q_{t}
$$

with $t=q_{t}^{2}$. To simplify the calculations we approximate

$$
\Gamma\left(b_{t}\right)=\frac{1}{R^{2}} e^{-\frac{b_{t}^{2}}{R^{2}}} .
$$

Intergrating over $r_{\perp}$ in Eq. (4) (see Ref. [6]) we have

$$
A\left(b_{t}, Q, x\right)=C \int_{0}^{1} d z 2\left\{1-Y\left(\kappa\left(x, b_{t}\right)\right)\right\} \Psi^{V}(0, z)
$$

where

$$
Y(\kappa)=\frac{1}{\kappa} e^{\frac{1}{\kappa}} E_{1}\left(\frac{1}{\kappa}\right)
$$

with

$$
\kappa\left(x, b_{t}\right)=\frac{2 \pi \alpha_{\mathrm{S}}}{3 a^{2} R^{2}} e^{-\frac{b_{t}^{2}}{R^{2}}} x G\left(x, a^{2}\right),
$$

$a^{2}=Q^{2} z(1-z)+m_{Q}^{2}$, and $m_{Q}$ denotes the quark mass. 
In general, the slope of the differential cross section at $\mathrm{t}=0$, is given by:

$$
B=\frac{1}{2} \frac{\int A\left(b_{t}, Q, x\right) b_{t}^{2} d^{2} b_{t}}{\int A\left(b_{t}, Q, x\right) d^{2} b_{t}}
$$

while the t-dependence of the differential cross section is obtained from

$$
\frac{\frac{d \sigma}{d t}}{\left.\frac{d \sigma}{d t}\right|_{t=0}}=\left|\frac{\int J_{0}\left(b_{t} \sqrt{|t|}\right) A\left(b_{t}, Q, x\right) d^{2} b_{t}}{\int A\left(b_{t}, Q, x\right) d^{2} b_{t}}\right|^{2}
$$

For the slope at $t=0$, we can derive a simple formula based on Eq. (8), namely

$$
B=\frac{R^{2}}{2} \frac{\int_{0}^{1} d z \int_{0}^{\infty} d u \frac{e^{-u}}{u} \ln (1+u \kappa) \Psi_{V}(z, 0)}{\int_{0}^{1} d z E_{1}\left(\frac{1}{\kappa}\right) e^{\frac{1}{\kappa}} \Psi_{V}(z, 0)} .
$$

In Figs.1 and 2 we plot the values that we obtain for the slope $B$, for different values of $Q^{2}$, as a function of the energy, where we have taken $R^{2}=6 \mathrm{GeV}^{-2}$. For $\mathrm{J} / \psi$ production we have $z=1 / 2$ ( see Ref. [6] for details), while for $\rho$ production we need to integrate over $z$ using the asymptotic wave function of the form $\Psi_{\rho}(z, 0)=z(1-z) \phi_{\rho}(0)$.

\subsection{Two radii approach}

Recent data from HERA on $\mathrm{J} / \psi$ production 14 has some novel features, one is the fact that the elastic and inelastic photo and lepto produced $\mathrm{J} / \psi$, have completely different slopes ( $t$-dependence) with $B_{e l}=4 \mathrm{GeV}^{-2}$ and $B_{\text {in }}=1.66 \mathrm{GeV}^{-2}$ (see Fig. 3), although the values of the integrated cross section are the same. This suggests that two different radii maybe present in the proton target. Consequently, we extend our formalism to a two radii model, with the aid of a generating function

$$
\sigma\left(\eta, x, r_{\perp}^{2} ; b_{t}\right)=\sum_{n=0}^{\infty}(-1)^{n} e^{-n \eta} C_{n}\left(x, r_{\perp}^{2} ; b_{t}\right)
$$

where $C_{n}$ denotes the amplitude for $n$ - Pomeron exchange. For vector meson production the amplitude is:

$$
A\left(x, Q^{2} ; b_{t}\right)=C \int \frac{d^{2} r_{\perp}}{\pi} \int_{0}^{1} d z \Psi_{\gamma^{*}}\left(Q, z, r_{\perp}\right) \sigma\left(\eta=0, x, r_{\perp} ; b_{t}\right) \Psi_{V}\left(r_{\perp}=0, z\right) .
$$

A trivial calculation shows that Eq. (四) is a solution of the equation

$$
e^{-\eta} \frac{d \sigma}{d \eta}=\kappa(1-\sigma)
$$


as illustrated in Fig. Ha. For the two radii model we obtain a set of equations which are illustrated in Figs. 4lb-d.

$$
\begin{gathered}
e^{-\eta} \frac{d \sigma_{e}^{e}}{d \eta}=\kappa_{1}(1-\sigma)-\kappa_{2} \sigma_{e}^{i} ; \\
e^{-\eta} \frac{d \sigma_{e}^{i}}{d \eta}=\kappa_{2}\left(1-\sigma_{i}^{i}\right)-\kappa_{1} \sigma_{e}^{i} ; \\
e^{-\eta} \frac{d \sigma_{i}^{i}}{d \eta}=\kappa_{2}\left(1-\sigma_{i}^{i}\right) .
\end{gathered}
$$

In Eq. (16) we have assumed the same values of $\kappa$ for a transition of a proton to inelastic state, and for an inelastic state to an inelastic state. This is true in the additive quark model and we consider it a reasonable first approximation here. $\kappa_{1}$ and $\kappa_{2}$ are defined as follows:

$$
\begin{aligned}
\kappa_{1}\left(x, b_{t}\right) & =\frac{2 \pi \alpha_{\mathrm{S}}}{3 a^{2} R_{1}^{2}} e^{-\frac{b_{t}^{2}}{R_{1}^{2}}} x G\left(x, a^{2}\right) ; \\
\kappa_{2}\left(x, b_{t}\right) & =\frac{2 \pi \alpha_{\mathrm{S}}}{3 a^{2} R_{2}^{2}} v e^{-\frac{b_{t}^{2}}{R_{2}^{2}}} x G\left(x, a^{2}\right) ;
\end{aligned}
$$

with $v=\frac{R_{1}}{R_{2}}$ whose value is taken from the experimental data, which shows the same cross section for elastic and inelastic diffractive production of $\mathrm{J} / \psi$ meson [14].

The solution to Eq. (16) is

$$
\begin{gathered}
\sigma_{e}^{e}\left(\eta=0, x, r_{\perp} ; b_{t}\right)=1-e^{-\kappa_{1}}+\frac{\kappa_{2}^{2}}{\kappa_{1}-\kappa_{2}} e^{-\kappa_{1}}+ \\
\frac{\kappa_{2}^{2}}{\left(\kappa_{1}-\kappa_{2}\right)^{2}}\left(e^{-\kappa_{1}}-e^{-\kappa_{2}}\right)
\end{gathered}
$$

and

$$
\sigma_{e}^{i}\left(\eta=0, x, r_{\perp} ; b_{t}\right)=\frac{\kappa_{2}}{\kappa_{1}-\kappa_{2}}\left\{e^{-\kappa_{2}}-e^{-\kappa_{1}}\right\}
$$

Using the technique developed in Ref. [6] we can integrate over $r_{\perp}$ and obtain:

$$
\begin{gathered}
A_{e}^{e}\left(x, Q^{2} ; b_{t}\right)=C \int_{0}^{1} d z\left\{1-Y\left(\kappa_{1}\right)-\frac{\kappa_{2}^{2}}{\kappa_{1}\left(\kappa_{1}-\kappa_{2}\right)}\left[\frac{1}{\kappa_{1}}\left(1-Y\left(\kappa_{1}\right)\right)-Y\left(\kappa_{1}\right)\right]-\right. \\
\left.\frac{\kappa_{2}^{2}}{\left(\kappa_{1}-\kappa_{2}\right)^{2}}\left[Y\left(\kappa_{2}\right)-Y\left(\kappa_{1}\right)\right]\right\} \frac{\Psi_{V}(0, z)}{a^{2}},
\end{gathered}
$$

and

$$
A_{e}^{i}\left(x, Q^{2} ; b_{t}\right)=C \int_{0}^{1} d z \frac{\kappa_{2}}{\kappa_{1}-\kappa_{2}}\left\{Y\left(\kappa_{1}\right)-Y\left(\kappa_{2}\right)\right\} \frac{\Psi_{V}(z, 0)}{a^{2}} .
$$




\section{Results and Discussion}

The most important parameters in our formalism are $\kappa_{i}$ (with $\mathrm{i}=1,2$, see Eq. (20)), they depend on the form assumed for the profile function $\Gamma\left(b_{t}\right)$, and on the value of the gluon density $x G\left(x, Q^{2}\right)$. Our formalism for the $\mathrm{SC}$ only includes screening due to the propagation of the $\bar{q} q$ pair through the target. We assume that the $\mathrm{SC}$ in the gluon sector, have been incorporated in the parametrization used for the gluon density, which has been determined by fitting to the experimental data. In all our calculations we have used the GRV parametrization [18], which successfully describes the experimental data on $F_{2}\left(x, Q^{2}\right)$ down to small photon virtualities, $Q^{2} \approx 1.5 \mathrm{GeV}^{2}$.

Our parametrization depends on the values taken for the radii $R_{1}^{2}$ and $R_{2}^{2}$, which describe the $\mathrm{t}\left(b_{t}\right)$ dependence of the two gluon form factor at the elastic and inelastic proton vertex. We have chosen $R_{1}^{2}=6 \mathrm{GeV}^{-2}$ and $R_{2}^{2}=2 \mathrm{GeV}^{2}$, these values reproduce the experimental data for the t-slope in elastic and inelastic photoproduction of $J / \psi$ $(\gamma p \rightarrow J / \psi p)$ and $(\gamma p \rightarrow J / \psi X)$ i.e. $B_{e l}=4.0 \pm 0.3 \mathrm{GeV}^{-2}$ and $B_{i n}=1.6 \pm 0.3 \mathrm{GeV}^{-2}$ at $\mathrm{W}=100 \mathrm{GeV}$ (see [20]). For $J / \psi$ production, typical transverse distances are of the order $r_{\perp}^{2} \approx\left(\frac{Q^{2}}{4}+m_{c}^{2}\right)^{-1}$, which justifies the use of pQCD. For this reason we used the $J / \psi$ diffractive data to fix our parameters. Our results for $J / \psi$ are plotted in Fig.1, which displays the $\mathrm{W}$ and $Q^{2}$ dependence of the effective slope. As expected, SC change the dependence of $B$ on the kinematical variable. For example, for $\mathrm{W}=100 \mathrm{GeV}$ and $Q^{2}=$ 0 , the slope is $\mathrm{B}=4 \mathrm{GeV}^{-2}$, while at large $Q^{2}\left(Q^{2}=50 \mathrm{GeV}^{2}\right)$ and the same energy, the slope decreases to a value $\mathrm{B}=3.1 \mathrm{GeV}^{-2}$. The energy dependence of the slope can be parametrized by an effective $\alpha_{\text {eff }}^{\prime}$, using Eq. (2) we can write the slope in the form

$$
B=B_{0}+4 \alpha_{e f f}^{\prime} \ln \left(\frac{W}{W_{0}}\right)
$$

For $Q^{2}=0$, in the energy range $50 \leq \mathrm{W} \leq 500 \mathrm{GeV}$, we obtain a value $\alpha_{\text {eff }}^{\prime}=0.08 \mathrm{GeV}^{-2}$. For large values of $Q^{2}, \alpha_{e f f}^{\prime}$ decreases. Our value for the slope at $Q^{2}=0$, is approximately one fourth of the conventional value assumed for the slope of the soft Pomeron.

We wish to stress, that both experimental and theoretical values quoted for the slope are strongly dependent on the manner in which the t-slope, is determined. To investigate this ambiguity, we have calculated the $t$ dependence of the differential cross section for $J / \psi$ production for different intervals of $t$. In Fig.5 we clearly see the effect of the SC in changing the exponential dependence in t. It gives rise to a positive curvature at small $\mathrm{t}$, and a dip at $|t| \approx 1 \mathrm{GeV}^{2}$. The position of the dip is energy dependent, and at high energies the dip moves to smaller values of $|t|$. One should therefore take heed when attempting to determine the slope from measurements made over a wide range of t. For 
example, for $Q^{2}=0$ and $\mathrm{W}=50 \mathrm{GeV}$ we obtain a $B_{\text {eff }}=4.6 \mathrm{GeV}^{-2}$, when determining the slope over the range from $\mathrm{t}=0$ to $|t|=0.8 \mathrm{GeV}^{-2}$. This is to be compared with $B_{\text {eff }}$ $=3.9 \mathrm{GeV}^{-2}$ at $\mathrm{t}=0$ (see Fig.1). The energy dependence of the slope determined from the $0 \leq|t| \leq 0.8 \mathrm{GeV}^{2}$ distribution gives a $\alpha_{e f f}^{\prime}=0.19 \mathrm{GeV}^{-2}$, which is close to the value for the soft Pomeron.

The t slope, the scale of the effective slope, as well as $\alpha_{\text {eff }}^{\prime}$ depend on the values taken for the radii. This is illustrated in Figs.5, 2 and 0 . These quantities also differ for the one and two radii models. Taking $R_{1}^{2}=10 \mathrm{GeV}^{-2}$ and $R_{2}^{2}=3 \mathrm{GeV}^{-2}$ produce a minimum at values of $|t|<1 \mathrm{GeV}^{2}$.

For $\rho$ production the case is more complicated, as we are not sure that we are dealing only with a hard process [14. Our results for $\rho$ production are qualitatively the same as those for $\mathrm{J} / \psi$ (see Fig. 2). However, for $\rho$ production we fail to reproduce the experimentally measured value of $\mathrm{B}=6 \mathrm{GeV}^{-2}$ ( at $Q^{2}=5-10 \mathrm{GeV}^{2}$ ), as we obtain a value $\mathrm{B}=4 \mathrm{GeV}^{-2}$. This is not suprizing, as our approach is based on pQCD, and in $\rho$ production for values of $Q^{2}=5-10 \mathrm{GeV}^{2}$, we still expect a non-perturbative component, which we have not taken into account, to present. At larger values of $Q^{2}$ (where one can rely exclusively on pQCD) the experimental slope [21] approaches the value $\mathrm{B}=3-4 \mathrm{GeV}^{-2}$. We find that $\alpha_{\text {eff }}^{\prime}$ is small in the region of large $Q^{2}\left(\approx 0.05 \mathrm{GeV}^{-2}\right.$ at $\left.\mathrm{t}=0\right)$.

In Eq. (2), we have parametrized the profile function $\Gamma\left(b_{t}\right)$ by an exponential form. For small $t$ where our result is rather insensitive to the form taken for $\Gamma\left(b_{t}\right)$, the exponential form is adequate. At larger $|t|$ this is no longer so. We have checked the influence of taking a different form for $\Gamma\left(b_{t}\right)$ on the t-distribution. For the case where

$$
\Gamma\left(b_{t}\right)=\frac{2}{R^{2}}\left(2 \sqrt{2} \frac{b_{t}}{R}\right) K_{1}\left(2 \sqrt{2} \frac{b_{t}}{R}\right)
$$

(where $K_{1}(x)$ denotes the associated Bessel function of first order), this corresponds to assuming a dipole for the two gluon form factor

$$
F(t)=\left(1+\frac{R^{2}|t|}{8}\right)^{-2}
$$

We find that there is no change in the energy dependence of the slope at $t=0$. However, the dipole form factor does effect the t distribution for $|t| \geq 0.3 \mathrm{GeV}^{2}$, making it shallower than the one obtained with an exponential form factor. Our result that $\alpha_{\text {eff }}^{\prime} \approx \alpha_{P}$ (soft Pomeron) over a wide range of t, still holds.

We notice that the power -like form factor, given in the previous equation, leads to a slope which depends strongly on the region of $t$ where the slope is measured. Indeed, 
using this equation we have

$$
B\left(t=t_{0}\right)=\frac{R^{2}}{2\left(1+\frac{R^{2}}{8}\left|t_{0}\right|\right)} .
$$

With $\left|t_{0}\right|=0.1 G e V^{2}$ and $R^{2}=10 G e V^{-2}$ we have $B(|t|=0.1)=0.8 B(t=0)$. Therefore, part of the $Q^{2}$ dependence of the experimental slope, could be attributed to the different values of $t_{\text {min }}$, at which the slope was measured.

\section{Conclusions}

1. Based on the new experimental data from HERA [14], we have assumed a two radii model for the proton. We have obtained formulae for shadowing screening corrections for diffractive vector meson production in DIS. This has been calculated within the framework of the DGLAP evolution equations for the low $x$ region.

2. The influence of the $\mathrm{SC}$ on the absolute value, the $Q^{2}$ and $\mathrm{W}$ dependence of the tslope for diffractive vector production in DIS, is discussed. We show that SC induces a shrinkage of the $t$ slope. We also calculated the value of the effective $\alpha^{\prime}$.

3. We summarize the results of our calculations in Table I, which shows the values of the intercept and the slope of the effective trajectory of the hard Pomeron, that we obtain from our calculations. Comparing the trajectories of the hard Pomeron obtained without and with SC, we note that the SC reduces the value of the intercept and generates an effective $\alpha_{e f f}^{\prime}$ for the hard Pomeron.

4. We show that the $\mathrm{SC}$ changes the t-distribution, creating a positive curvature at small values of $t$, and a dip at $|t| \approx 1 \mathrm{GeV}^{2}$. These two phenomena suggest, that one should be careful when attempting to determine the t-slope from data taken over a wide range of t. The slope determined under such conditions, suggest a shrinkage which is much larger than the slope at $\mathrm{t}=0$, given in Table $\mathrm{I}$.

5. We have chosen the values of the two radii to reproduce the experimental data for $J / \psi$ diffractive production, where one can safely apply pQCD. However, these values fail to reproduce the value of the t-slope for $\rho$ production in the region of $Q^{2}=5$ $10 \mathrm{GeV}^{2}$. The results of our phenomenological investigation support the conclusions of the $\mathrm{H} 1$ collaboration [14, in that hard physics effects already dominate at very small $Q^{2}$ for $\mathrm{J} / \psi$ production, while for $\rho$ production even at values of $Q^{2}=10-15 \mathrm{GeV}^{2}$, a non negligible soft component still appears to be present. Direct evidence for this is the 
experimentally observed shrinkage of the forward elastic peak for $\rho$ production, which is much larger than that expected for only hard processes [7] (even when SC are included).

6. Based on our investigation we recommend that:

(i) The diffractive t-slope be determined from measurements made for $|t| \leq 0.3 \mathrm{GeV}^{2}$.

(ii) For $\rho$ production the hard diffractive slope should be measured for $Q^{2} \geq 15 \mathrm{GeV}^{2}$, where we expect $B_{\text {diff }} \approx 4(\mathrm{GeV})^{-2}$, or smaller.

\section{References}

[1] M.G. Ryskin: Z. Phys. C57 (1993) 89.

[2] B.Z. Kopeliovich et.al.: Phys. Lett. B324 (1994) 469.

[3] S.J. Brodsky et.al.: Phys. Rev. D50 (1994) 3134.

[4] ZEUS Collaboration; M.Derrick et al.: Phys. Lett. B350 (1996) 120; J Bulmann for the Collaboration: pa02-028,submitted to XXVIII Int. Conference on HEP Warsaw, June $25-31,1996$.

[5] H1 Collaboration; S.Aid et al.: Nucl. Phys. B468 (1996) 3; J. Gayler for the Collaboration:pa02-088,pa02-064,pa02-066,pa02-86 submitted to XXVII Int. Conference on HEP Warsaw, July 25-31,1996

[6] E. Gotsman, E.M. Levin and U. Maor: Phys. Lett. B353 (1995) 526.

[7] L. Frankfurt, W. Koepf and M.Strikman: Phys. Rev. D54 (1996) 3194.

[8] M.G.Ryskin et al.:DTP/95/96,hep-ph 9511228.

[9] A.Donnachie and P.V.Landshoff: Nucl. Phys. B244 (1984) 322; Nucl. Phys. B276 (1986) 690;

[10] M.M.Block,K.Kang and A.R.White: Northwestern University Preprint No.172 (1992).

[11] T.T.Chou and Chen Ning Yang: Phys. Lett. B244 (1990) 113.

[12] C.Bourelly,J.Soffer and T.T.Wu: Z. Phys. C37 (1988) 369.

[13] E. Gotsman, E.M. Levin and U. Maor: Phys. Lett. B309 (1993) 109; Z. Phys. C57 (1993) 667; Phys. Rev. D49 (1994) R4321; Phys. Lett. B347 (1995) 424 
[14] H1 Collaboration; S.Aid et al.: Nucl. Phys. B472 (1996) 3; ZEUS Collaboration; M.Derrick et al.: Phys. Lett. B350 (1995) 120.

[15] Nucl. Phys. B231 (1989) 189; Phys. Lett. B296 (1992) 227. V.N. Gribov and L.N. Lipatov: Sov. J. Nucl. Phys.15(1972)438; L.N. Lipatov: Yad. Fiz.20 (1974) 181; G. Altarelli and G. Parisi: Nucl. Phys. B126 (1977) 298; Yu.L.Dokshitzer: Sov.Phys. JETP 46 (1977) 641.

[16] E.M. Levin and M.G.Ryskin: Sov. J. Nucl. Phys. 45 (1987) 150.

[17] A.H. Mueller: Nucl. Phys. B335 (1990) 115.

[18] M. Gluck, E. Reya and A. Vogt: Z. Phys. C53 (1992) 127.

[19] E.M. Levin and M.G. Ryskin: Phys.Rep. 189 (1990) 267.

[20] H1 Collaboration; S.Aid et al.: DESY -96-037, March 1996.

[21] H.Abramowicz: Rapporteur talk in Proceedings of XXVIII ICHEP Warsaw, July 25 -31, 1996, TAUP 2396-96, hep-ex 9612001. 


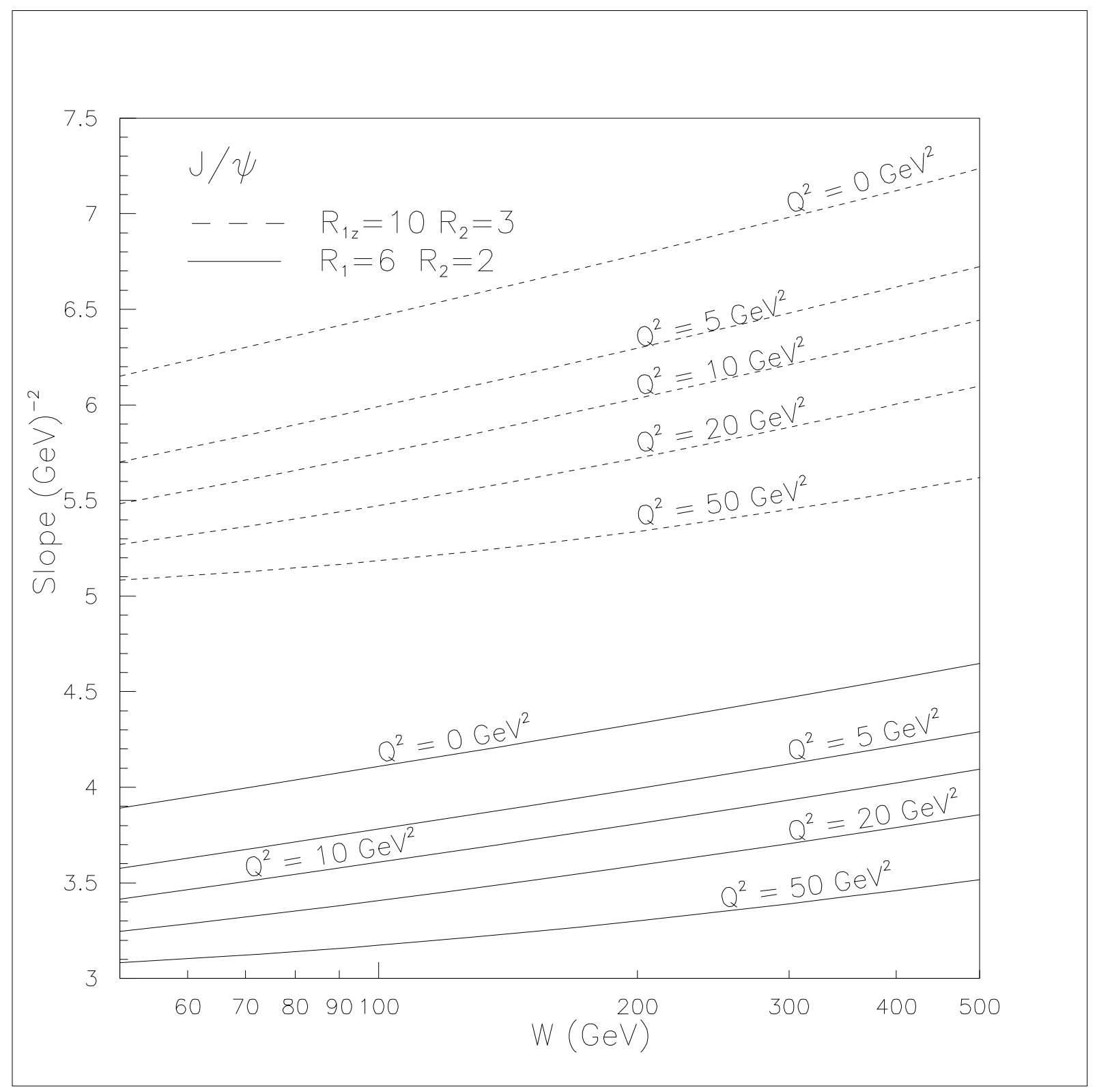

Figure 1: $W$ and $Q^{2}$ behaviour of the slope at $t=0$ for $\mathrm{J} / \psi$ diffractive production, for the two sets of radii. 


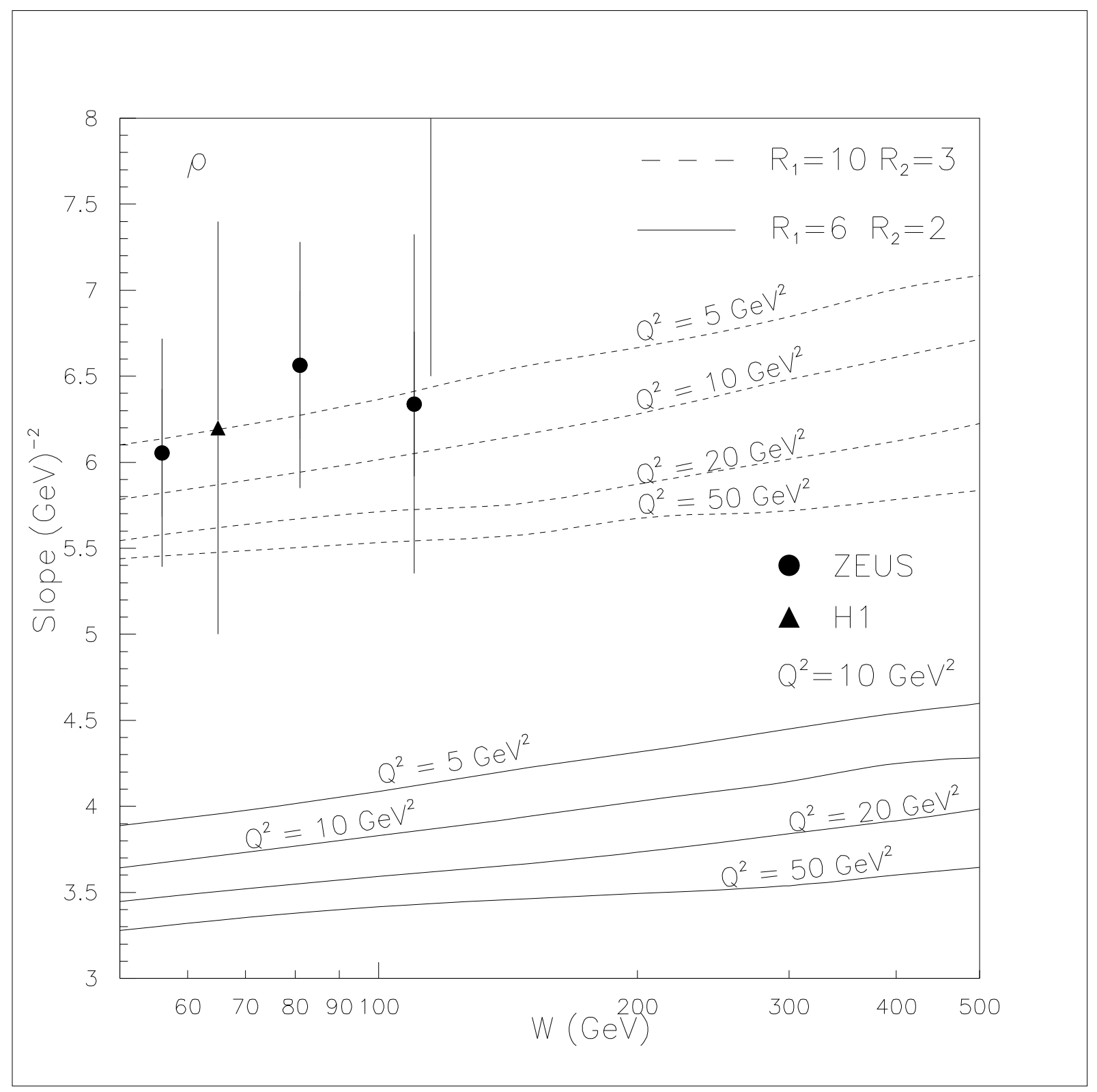

Figure 2: $W$ and $Q^{2}$ dependence of the slope at $t=0$ for $\rho$ diffractive production, for the two sets of radii. 


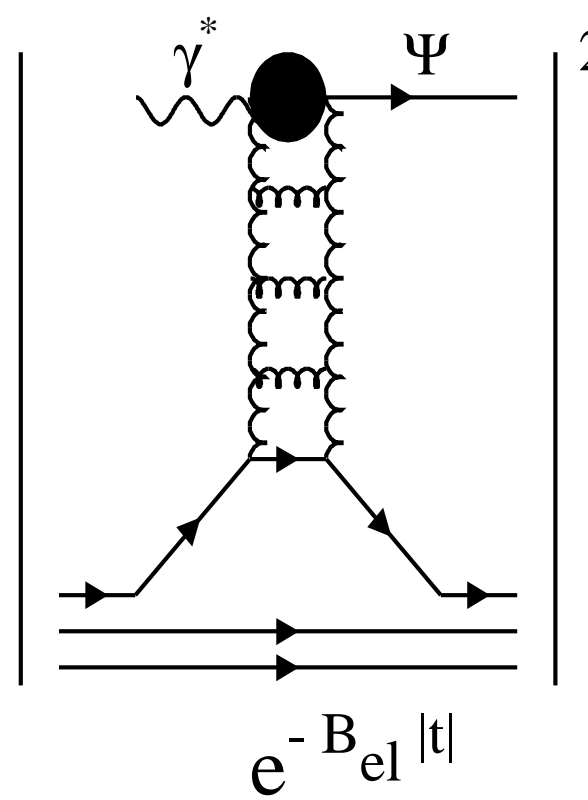

a )

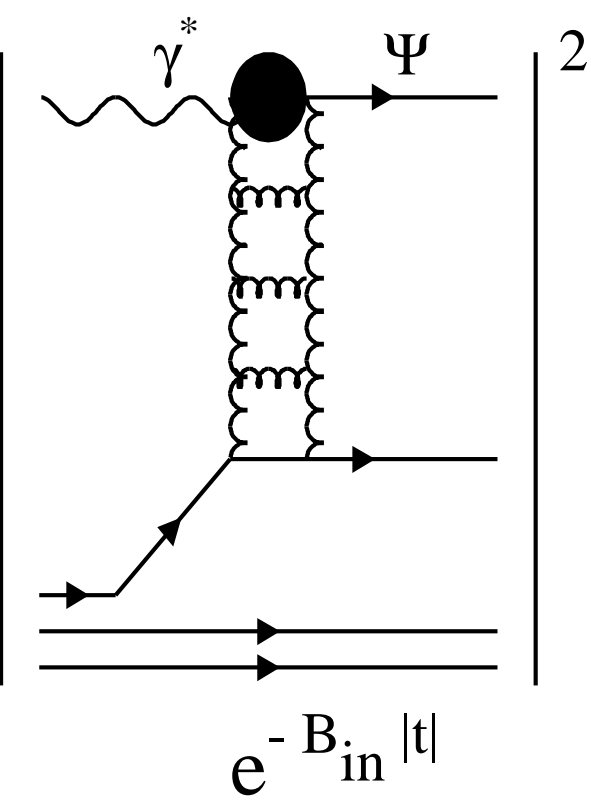

b)

Figure 3: The $\mathrm{J} / \psi$ production without (a) and with ( b) proton dissociation. 


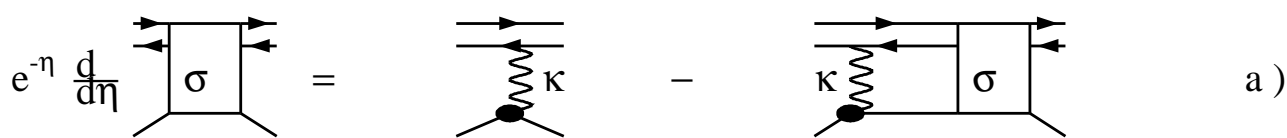

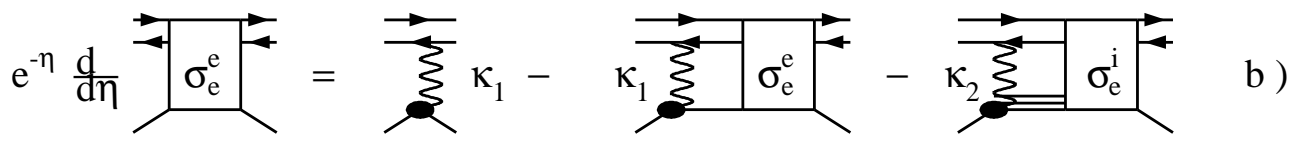

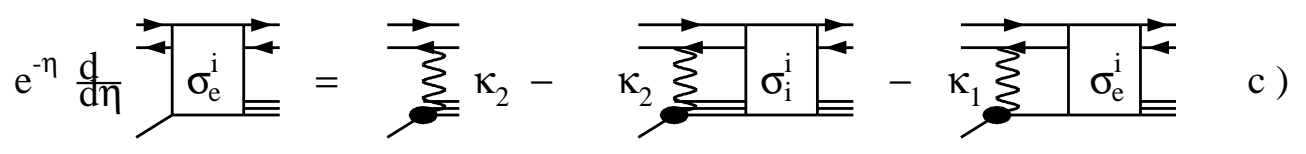

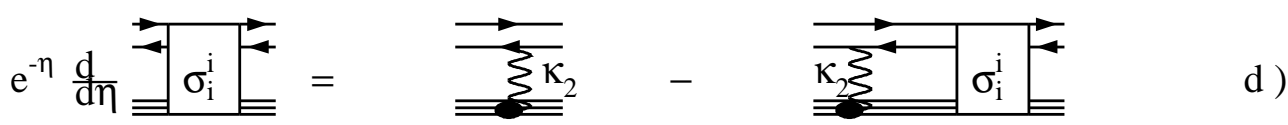

Figure 4: Equations for the SC in eikonal approach in the two radii model of the target proton. 


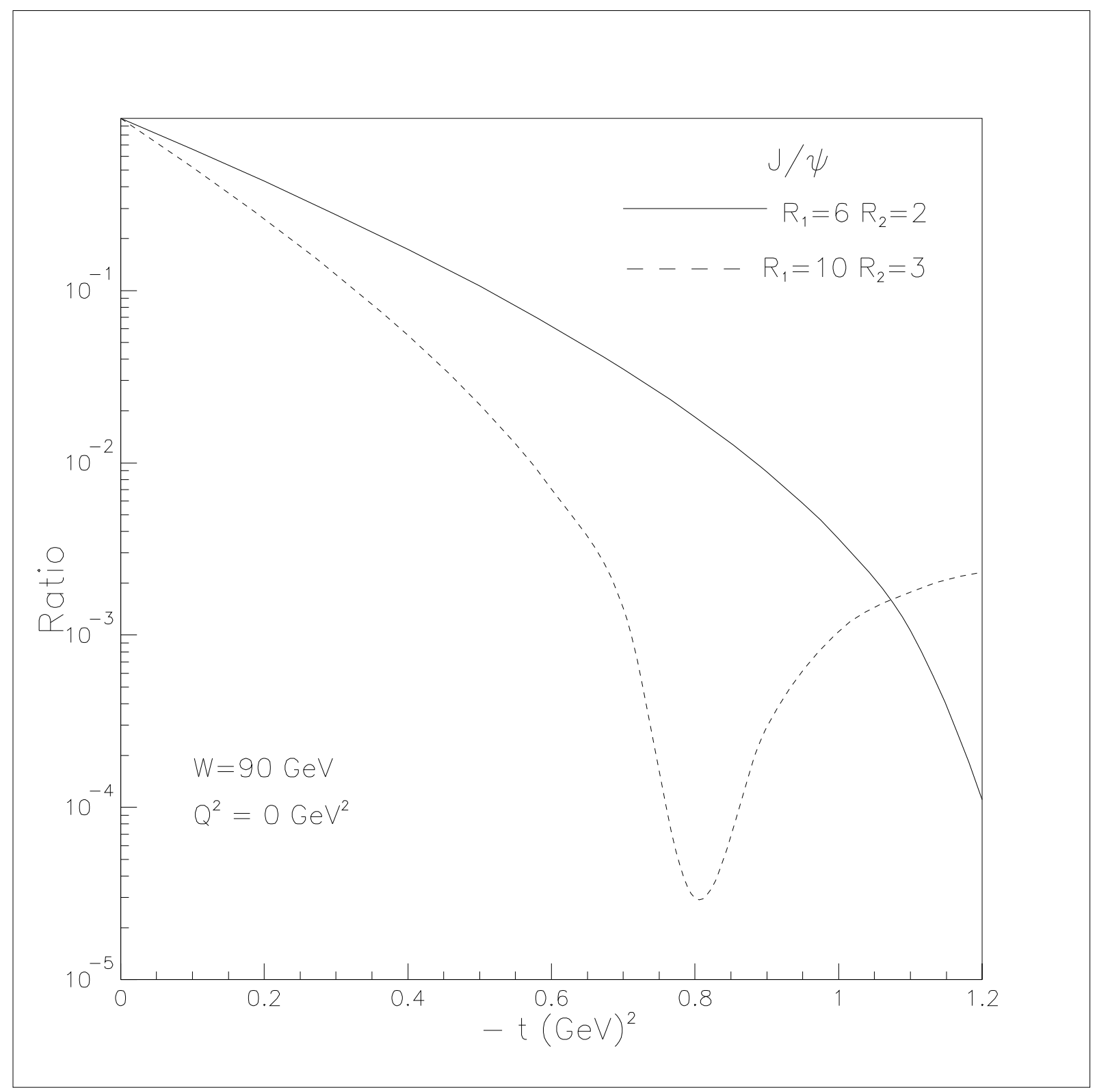

Figure 5: $t$-dependence of the differential cross section for diffractive $\mathrm{J} / \psi$ production. 


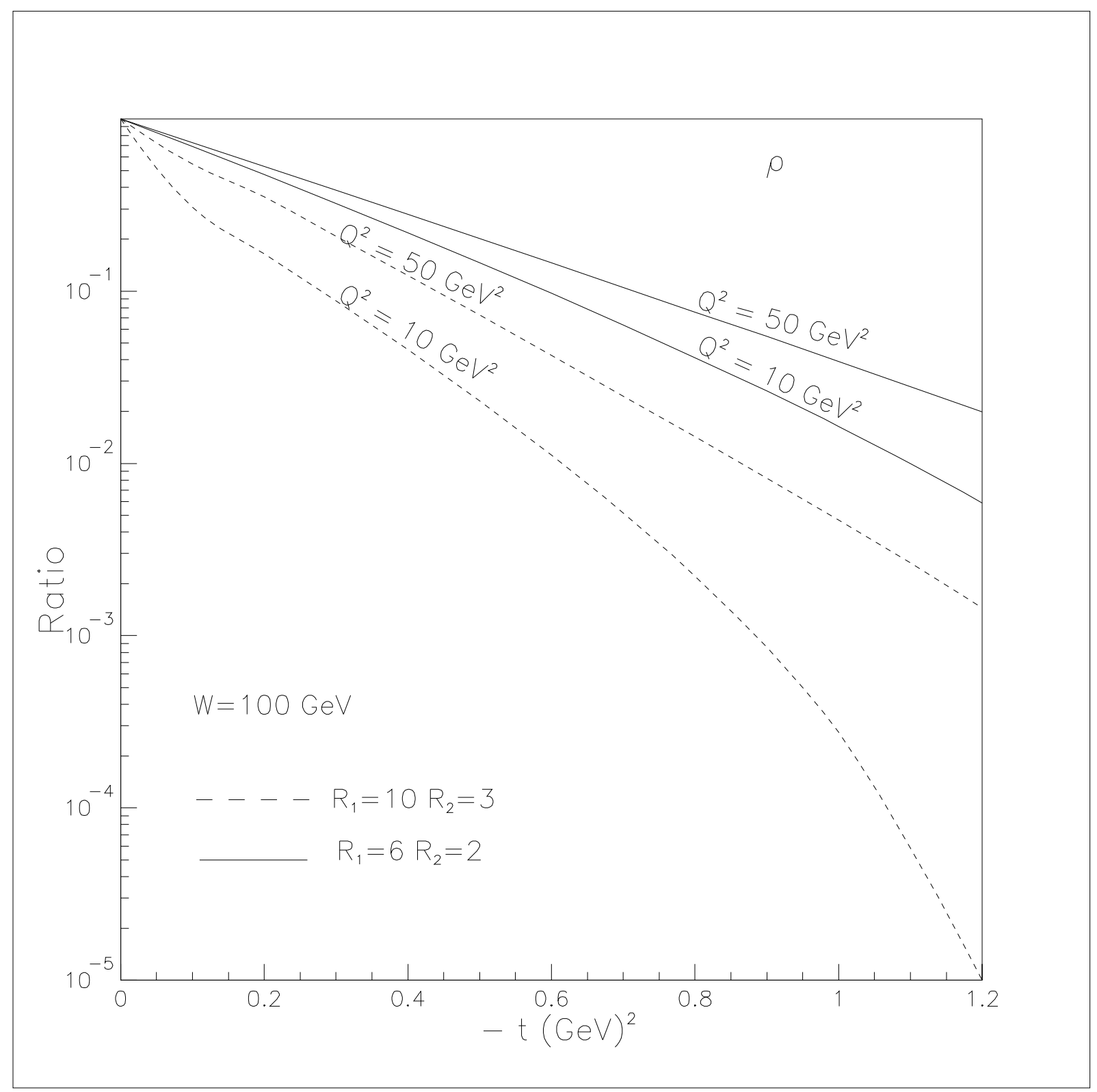

Figure 6: $t$-dependence of the differential cross section for diffractive $\rho$ production. 


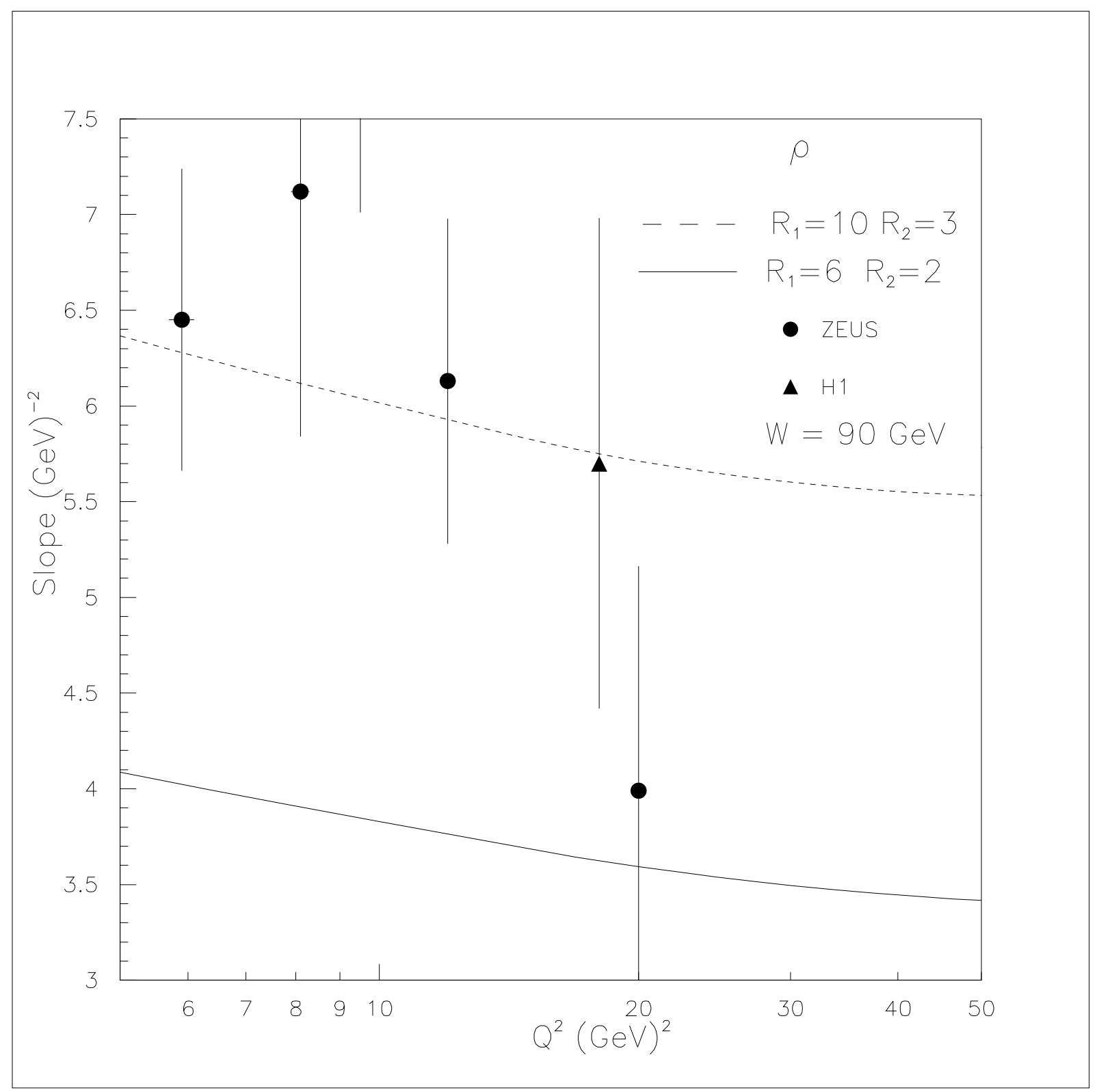

Figure 7: $Q^{2}$ dependence of the slope at $t=0$ for $\rho$ diffractive production. 
Table 1:

Parameters of the effective "hard" Pomeron trajectory $\alpha_{P}^{e f f}(t)=1+\epsilon+\alpha_{e f f}^{\prime} t$ in the GRV parametrization for the gluon structure function $\left(Q^{2}\right.$ in $G e V^{2}, \alpha_{e f f}^{\prime}, R_{i}^{2}$ in $\left.G_{e V}^{-2}\right)$.

\begin{tabular}{|c|c|c|c|c|c|c|c|c|c|c|c|c|}
\hline & \multicolumn{6}{|c|}{$\mathrm{J} / \Psi$} & \multicolumn{6}{|c|}{$\rho$} \\
\hline \multirow[t]{2}{*}{$Q^{2}$} & \multicolumn{2}{|c|}{ without SC } & \multicolumn{2}{|c|}{$R_{1}^{2}=6, R_{2}^{2}=2$} & \multicolumn{2}{|c|}{$R_{1}^{2}=10, R_{2}^{2}=3$} & \multicolumn{2}{|c|}{ without SC } & \multicolumn{2}{|c|}{$R_{1}^{2}=6, R_{2}^{2}=2$} & \multicolumn{2}{|c|}{$R_{1}^{2}=10, R_{2}^{2}=3$} \\
\hline & $\epsilon$ & $\alpha_{e f f}^{\prime}$ & $\epsilon$ & $\alpha_{e f f}^{\prime}$ & $\epsilon$ & $\alpha_{e f f}^{\prime}$ & $\bar{\epsilon}$ & $\alpha_{e f f}^{\prime}$ & $\epsilon$ & $\alpha_{e f f}^{\prime}$ & $\bar{\epsilon}$ & $\alpha_{e f f}^{\prime}$ \\
\hline 0 & 0.32 & 0 & 0.21 & 0.076 & 0.23 & 0.135 & $\mathrm{~N} / \mathrm{A}$ & $\mathrm{N} / \mathrm{A}$ & $\mathrm{N} / \mathrm{A}$ & $\mathrm{N} / \mathrm{A}$ & $\mathrm{N} / \mathrm{A}$ & $\mathrm{N} / \mathrm{A}$ \\
\hline 5 & 0.38 & 0 & 0.27 & 0.06 & 0.29 & 0.114 & 0.2 & 0 & 0.15 & 0.08 & 0.17 & 0.11 \\
\hline 10 & 0.40 & 0 & 0.31 & 0.06 & 0.33 & 0.103 & 0.32 & 0 & 0.20 & 0.07 & 0.22 & 0.11 \\
\hline 20 & 0.45 & 0 & 0.37 & 0.06 & 0.38 & 0.08 & 0.38 & 0 & 0.27 & 0.06 & 0.28 & 0.07 \\
\hline 50 & 0.52 & 0 & 0.46 & 0.045 & 0.48 & 0.054 & 0.45 & 0 & 0.36 & 0.05 & 0.38 & 0.04 \\
\hline
\end{tabular}


